\title{
Role of TRAILR1 and TNFR1A polymorphisms in the susceptibility and pharmacogenetics of rheumatoid arthritis and ankylosing spondylitis patients treated with infliximab
}

\author{
M J Morales-Lara', P Conesa-Zamora ${ }^{2 *}$, V Santaclara², D Torres-Moreno ${ }^{2}$, F Pedrero $^{3}$, J Corral ${ }^{4}$, M Perez-Guillermo $^{2}$, \\ F J Rodríguez-Martínez ${ }^{5}$ E Soriano-Navarro ${ }^{5}$
}

From 5th European Workshop on Immune-Mediated Inflammatory Diseases

Sitges-Barcelona, Spain. 1-3 December 2010

\section{Introduction}

Polymorphisms in genes involved in antibody-dependent cellular cytotoxicity and apoptosis have been associated with inter-individual differences in the response to antiTNF agent infliximab in arthritides. TRAILR1 and $T N F R 1 A$ are two genes related to the extrinsic pathway of apoptosis.

\section{Aim}

To evaluate the role of two polymorphisms (rs20575/ G36A and rs767455/C626G) in these genes in the susceptibility and pharmacogenetics of rheumatoid arthritis (RA) and ankylosing spondylitis (AS) patients treated with infliximab. Patients and Methods. TRAILR1 and TNFR1 genotyping was performed in 138 patients (86 RA and $52 \mathrm{AS}$ ), and in two groups of controls (158 for RA and 182 for AS). A subset of 47 RA and 40 AS patients was also studied for the association of this polymorphism with the response to infliximab treatment assessed by EULAR and BASDAI criteria, respectively.

\section{Results}

No significant differences were observed between cases and controls in the genotype and allele distribution of TRAILR1 or TNFR1A polymorphisms for both diseases, though a tendency for a RA protective role of TNFR1A AA genotype was observed (29.8\% vs. $38.2 \%$; $\mathrm{p}=0.1172)$.
TRAILR1 G allele was associated with poor response after 3 month of infliximab treatment (G: $32.4 \%$ vs. $\mathrm{C} 14 \% ; \mathrm{p}=0.044$ ). Similar results were observed for AS, as the prevalence of non-responders was significantly lower in $\mathrm{C}$ carriers both, at 3 months $(21.4 \%$ vs. $83.3 \%$; $\mathrm{p}=0.003)$ and 6 months ( $11.5 \%$ vs. $50 \%$; $\mathrm{p}=0.029)$ of infliximab treatment. No significant differences were observed for TNFR1 polymorphism in terms of response.

\section{Conclusions}

This work provides the first evidence that rs 20575 polymorphism in TRAILR1 seems to influence the response to infliximab treatment both in RA and AS patients.

\section{Author details}

${ }^{1}$ Pharmacy Dept., SM Rosell University Hospital (HUSMR), Cartagena, Spain. ${ }^{2}$ Molecular Pathology and Pharmacogenetics Group FFIS011, HUSMR, Cartagena, Spain. ${ }^{3}$ Day Hospital, HUSMR, Cartagena, Spain. ${ }^{4}$ Centro Regional de Hemodonación, University of Murcia, Spain. ${ }^{5}$ Rheumatology Dept., HUSMR, Cartagena, Spain.

Published: 25 November 2010

\section{doi:10.1186/1479-5876-8-S1-P50}

Cite this article as: Morales-Lara et al:: Role of TRAILR1 and TNFR1A polymorphisms in the susceptibility and pharmacogenetics of rheumatoid arthritis and ankylosing spondylitis patients treated with infliximab. Journal of Translational Medicine 2010 8(Suppl 1):P50. 